\title{
PLANETARY NEBULAE AND HALO DYNAMICS IN EARLY TYPE GALAXIES
}

\author{
X. HUI \\ Astronomy Department, California Institute of Technology, USA \\ and \\ H.C. FORD \\ Department of Physics and Astronomy, The Johns Hopkins University \\ and Space Telescope Science Institute, USA
}

\section{Introduction}

The study of extragalactic planetary nebulae has made rapid progress in recent years with the help of high quantum efficiency detectors. A brief but distinctive phase in the late stage of stellar evolution, planetary nebulae ( $\mathrm{PNe}$ ) are not only interesting objects in their own right, but also are extremely valuable and unique tools for probing the host galaxies. Recent studies have used planetary nebulae as test particles to investigate the dynamics and mass distributions in the halos of early type galaxies.

In spiral galaxies HI rotation curves are measured far beyond the optically bright disks and are usually flat at large radii, indicating a dark halo whose mass is often several times that of the luminous matter. Because most early type galaxies contain very little gas, and individual stars are too faint to observe, information on the mass distributions and stellar kinematics in these galaxies is usually derived from integrated absorption spectra. However, the rapid falloff of surface brightness severly limits such measurements, and high signal-to-noise spectra are only possible in the bright central regions. Very little is known about the halos of early type galaxies.

Planetary nebula surveys have been carried out in over 20 early type galaxies (Ciardullo et al. 1989, Jacoby et al. 1990, Ciardullo et al. 1991, Hui et al. 1992a and Ford et al. 1992). By comparing the CCD images taken on the characteristic emission line [O III] $\lambda 5007$ and the adjacent continuum, a large number of planetary nebulae can be identified in individual galaxies up to $15 \mathrm{Mpc}$. The radial velocities of these planetaries can then be measured efficiently with a multifiber spectrograph. As an example, in NGC 5128, a nearby giant elliptical galaxy, over 400 planetary nebula ( $\mathrm{PN}$ ) velocities were measured out to a galactic radius of $20 \mathrm{kpc}$ (Hui et al. $1992 \mathrm{~b}$ ). Planetary nebulae thus provide a unique way to study the stellar dynamics and mass distributions in galactic halos. If the globular clusters are also observed, comparison of the stars and clusters will help clarify how the halos formed. 
In this paper we focus on the PN kinematics and the halo mass distribution in NGC 5128 , the best studied galaxy to date. We then briefly describe the continuing efforts to measure PN velocities in more distant galaxies such as NGC 4594, NGC 3379, and elliptical galaxies in the Virgo Cluster.

\section{NGC 5128}

NGC 5128 (also known as the radio source Centaurus A), which has a dust lane lying along its photometric minor axis, is the nearest (3.5 Mpc, Hui et al. 1992a) example of a galaxy merger. Graham (1979) used observations of the HII regions along the edge of the dust lane to show that the gas and dust are in a highly inclined, rotating disk. Graham proposed that the gas and dust came from a gas cloud or small galaxy which merged with NGC 5128. Subsequent observations of the disk $H_{\alpha}$ emission (Bland, Taylor \& Atherton 1987; Nicholson, Bland-Hawthorn \& Taylor 1992) and neutral hydrogen 21-cm emission (van Gorkom et al. 1990) confirmed the earlier findings and revealed that the dust lane is "the projection of a severely warped, thin disc of gas and dust". Wilkinson et al. (1985) used absorption spectra to show that the rotation of the main stellar body is around the photometric minor axis of the elliptical component, perpendicular to the disk rotation. These papers demonstrate the prescience of Baade and Minkowski's belief that NGC 5128 is two galaxies in collision ( $c f$. Sandage 1961).

However, because of the low surface brightness in the halo, previous studies were largely restricted to the observations of the bright stellar envelope and the ionized gas associated with the conspicuous dust lane. To obtain stellar kinematic information in the halo, we surveyed NGC 5128 for planetary nebulae. The observations were made on the CTIO 4-m telescope using the prime focus CCD camera (Hui et al. 1992a). Figure 1 shows the locations of our CCD fields. Along the photometric major and minor axes, the survey frames were placed on both sides of the galaxy in order to measure the halo rotation. The outermost halo fields are approximately $20 \mathrm{kpc}$ from the center of the galaxy.

Spectroscopic observations were made using multifiber spectrographs on both the AAT and CTIO 4-m (Hui et al. 1992b). Over 400 velocities were measured from the [O III] $\lambda 5007$ line. The PN velocities are typically accurate to $4 \mathrm{~km} \mathrm{~s}^{-1}$ for spectra obtained on AAT and $30 \mathrm{~km} \mathrm{~s}^{-1}$ at CTIO. The observed PNe cover the entire galaxy to a radius of $10 \mathrm{kpc}$ and extend along the photometric major axis to $20 \mathrm{kpc}$.

\subsection{KINEMATICS}

2.1.1 A schematic PN velocity field The PN spatial distribution as projected on the sky is shown in Figure 2, where the symbols correlate with the PN velocities (relative to the systemic velocity of the galaxy): a circle is used if a PN is approaching and a cross is used if it is receding; the size of the symbol scales with the velocity. To reduce the scatter of the intrinsic velocity dispersion, each nebular velocity is replaced by the mean velocity of its neighboring $\mathrm{PNe}$ within $1.5 \mathrm{kpc}$ radius. Therefore, the figure is in fact a representation of the mean PN velocity field. Also shown in the figure are the approximate isophotes of the galaxy (Dufour, et al. 1979). The semi-major axes of the ellipses vary from 1 to 4 effective radius $\left(r_{e}\right)$ with a step size of $1 r_{e}$. 


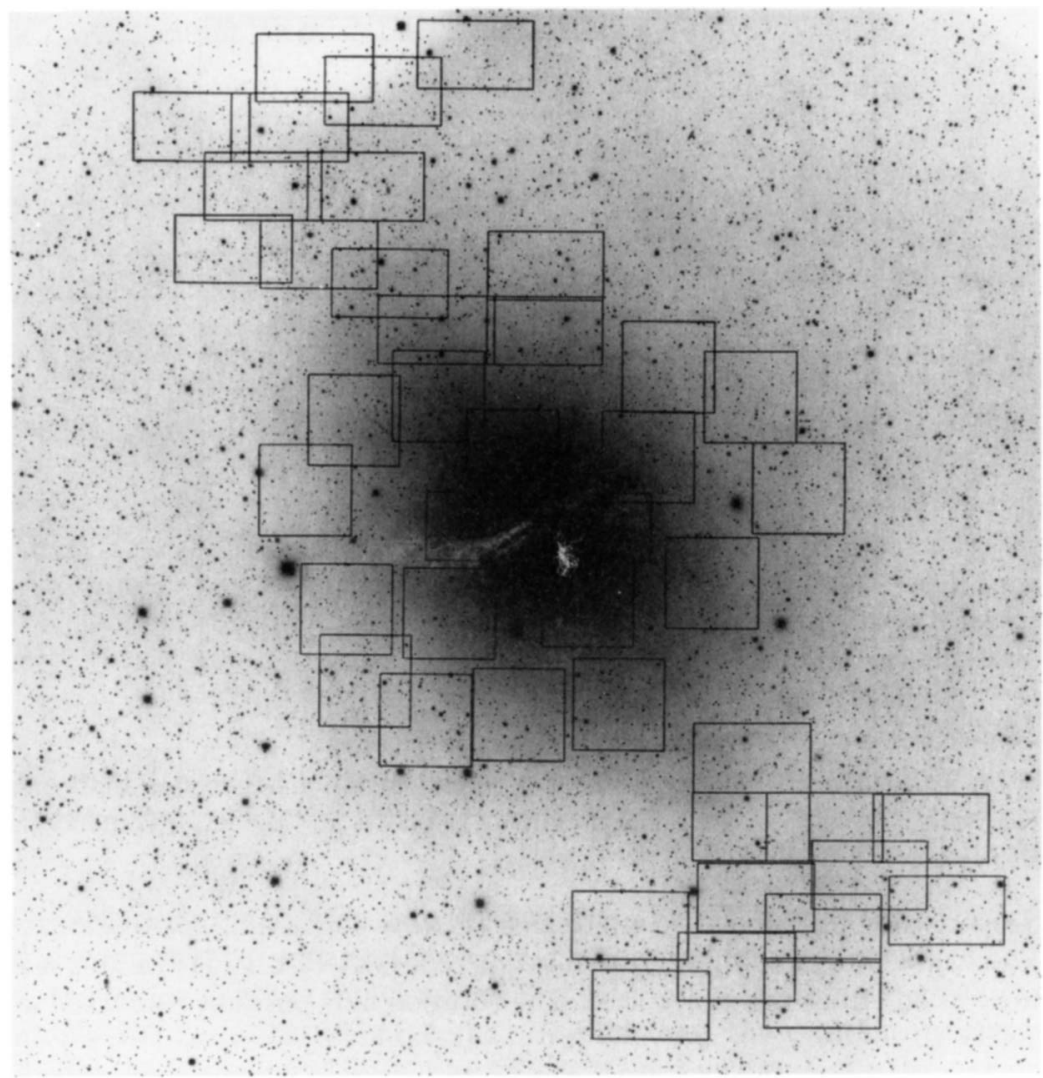

Figure 1. Each rectangle or square shows a CCD survey field. The center of the most distant fields are approximately $20 \mathrm{kpc}$ from the nucleus. North is at the top and the east at the left.

Several things can be seen in Figure 2. First, the nebulae apparently rotate around both the photometric major and minor axes. Second, the PN velocities extend to as far as $4 r_{e}$, a factor of 4 increase in radius compared to the observations of ellipticals made through integrated light. Finally, virtually no planetary nebula is found in the central $3 \times 6$ kpc region due to obscuration by the dust lane. The other blank areas in the distribution are due to the incomplete spatial coverage of our survey ( $c f$. Figure 1).

2.1.2 Rotation Axis Given the two dimensional PN velocity field, the rotation axis can be readily obtained by examining the velocity variation as a function of azimuthal angle. We fit the function

$$
V=A \times \sin \left(\Phi-\Phi_{0}\right)+V_{0}
$$

to individual $\mathrm{PN}$ velocities in a ring between 5 and $10 \mathrm{kpc}$ radius where the PN spatial coverage is most complete ( $c f$. Figure 2). In equation (1), $\Phi$ is the azimuthal angle on the 


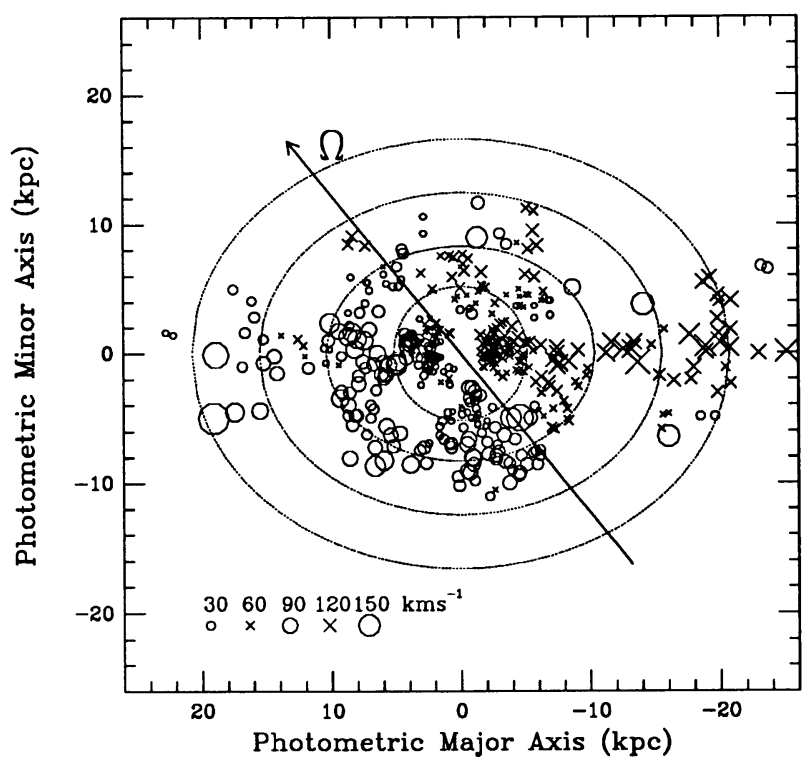

Figure 2. A schematic PN velocity field. The legend at lower left relates the symbol size to velocities (see text). The ellipses are the approximate isophotes and the letter $\Omega$ marks the position of the rotation axis.

sky with respect to the photometric minor axis $\left(\right.$ P.A. $\left.=305^{\circ}\right)$, and positive eastward. The best fit yields $\Phi_{0}=39^{\circ} \pm 10^{\circ}$. Thus, the rotation axis is at P.A. $=344^{\circ} \pm 10^{\circ}$, a significant misalignment of $39^{\circ}$ from the minor axis. The rotation axis position is marked in Figure 2 with the letter $\Omega$.

The large offset between the photometric and dynamical axes provides convincing evidence that NGC 5128 is not an axisymmetric system. Otherwise the rotation axis would fall either along the photometric minor axis (an oblate spheroid) or the photometric major axis (a prolate spheroid). Instead, the galaxy must be triaxial, in which case the observation can be explained naturally. Two effects can contribute to the misalignment. Generically, stars can rotate around both the intrinsic short axis or long axis in a triaxial potential ( $c f$. Statler 1987). Thus, the net angular momentum vector could be anywhere in the plane defined by the two. Alternatively, because the observed photometric axes are generally not the principal axes in projection for a triaxial ellipsoid, projection could also introduce misalignment even if the short axis is intrinsically the rotation axis (Stark 1977; Binney 1985).

2.1.3 Major and Minor Axis Velocity Profiles The major axis rotation is in the sense of $\mathrm{NE}$ approaching and SW receding, while the minor axis rotation is SE approaching and NW receding, the same as the dust lane. We construct the major axis rotation curve and velocity dispersion profile by folding the planetary nebulae in the NE onto the SW side. The PNe are binned in $2^{\prime}$ intervals inside $10^{\prime}$ and in $5^{\prime}$ intervals at larger radii. The mean velocity and its standard deviation yield a rotation velocity and a velocity dispersion for each bin. Similarly, the minor axis rotation curve and velocity dispersion profile are 


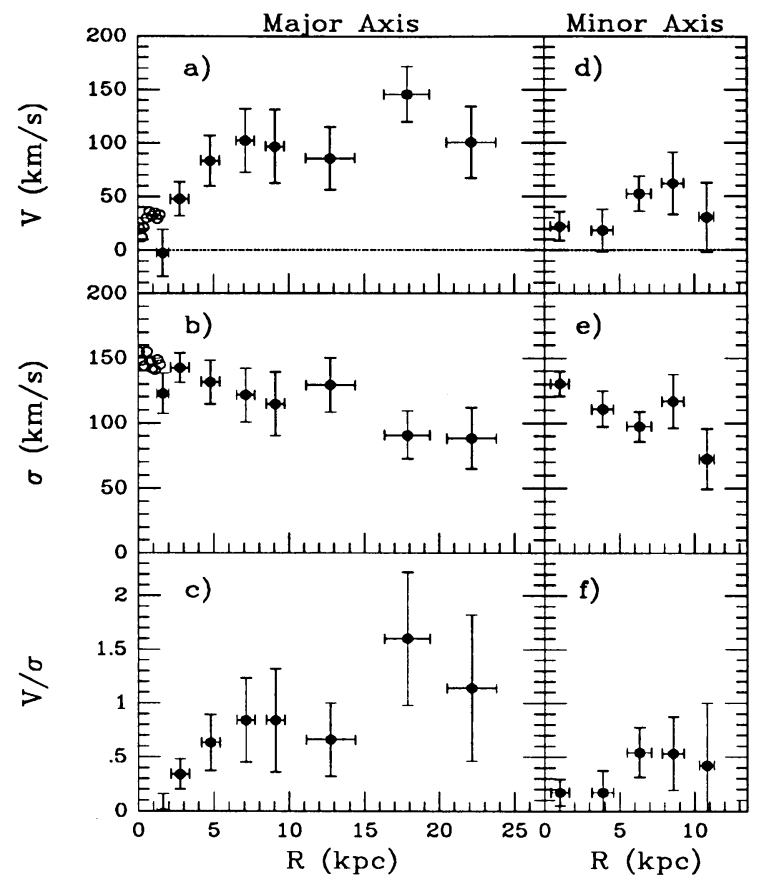

Figure 3. a) PN major axis rotation curve (black dots). Circles are the long slit data (Wilkinson et al. 1985). b) PN major axis velocity dispersion profile. Local $V / \sigma$ ratio along the major axis. d), e) and f) are the same but for the minor axis.

obtained by reflecting the SE side onto the NW side. The PNe are grouped in $2.5^{\prime}$ intervals. The resulting rotation curves and velocity dispersion profiles are shown in Figure 3.

Along the major axis, the rotation increases with radius in the inner galaxy and reaches approximately $100 \mathrm{~km} \mathrm{~s}^{-1}$ at about $7 \mathrm{kpc}$. Beyond that, the rotation is nearly flat. The velocity dispersion is about $143 \mathrm{~km} \mathrm{~s}^{-1}$ at $2.8 \mathrm{kpc}$, then declines slowly through out the halo to $\sim 90 \mathrm{~km} \mathrm{~s}^{-1}$ at $20 \mathrm{kpc}$. The streaming motion becomes more important with increasing radius. The local $V / \sigma$ ratio is approximately unity beyond $10 \mathrm{kpc}$ (Figure 3c). The minor axis rotation is about $50 \mathrm{~km} \mathrm{~s}^{-1}$ between 5 and $10 \mathrm{kpc}$. By comparing with the velocity dispersion, we find that the $V / \sigma$ ratio is about 0.5 for the minor axis rotation (Figure $3 \mathrm{f}$ ). Along the major axis, the PN rotation and velocity dispersion are consistent with those measured from the stellar absorption spectra (Wilkinson et al. 1985). However, planetary nebulae extend the measurement much further into the halo of the galaxy.

\subsection{MASS DISTRIBUTION OF THE GALAXY}

To derive the mass distribution of the galaxy, we apply a spherical Jeans equation to the major axis rotation and velocity dispersion:

$$
\frac{d\left(\rho \sigma^{2}\right)}{d r}=-\frac{G M(r) \rho}{r^{2}}+\frac{\rho V_{r o t}^{2}}{r}
$$

where the velocity dispersion is assumed to be isotropic. If the velocity dispersion in fact becomes more tangential with increasing radius, the isotropic model will overestimate the halo mass; on the other hand, the mass will be underestimated if the stellar orbits become more radial. 
Because the light distribution of the galaxy follows a de Vaucouleurs' law, we adopt the Hernquist mass model

$$
\rho(r)=\frac{M_{l} a}{2 \pi} \frac{1}{r(r+a)^{3}}
$$

where the scale length $a=r_{e} / 1.8153$. This model closely resembles de Vaucouleurs' law, yet allows dynamical properties of a galaxy to be evaluated analytically (Hernquist 1990; de Zeeuw 1990, private communication). The mass distribution corresponding to equation (3) can be written as

$$
M(r)=\frac{M_{l} r^{2}}{(r+a)^{2}}+\frac{M_{d} r^{2}}{(r+d)^{2}}
$$

with $M_{l}$ being the total mass of the potential. The second term is included to allow for dark matter. Although the Hernquist model has a finite total mass, it can approximate an isothermal halo when the scale length $d$ is sufficiently large (Dubinski \& Carlberg 1991).

To solve equation (2), we further assume that the intrinsic rotation curve is

$$
V_{\text {rot }}(r)=\frac{v_{0} r}{\left(r^{2}+r_{0}^{2}\right)^{1 / 2}}
$$

which is asymptotically flat at large radii $\left(r>r_{0}\right)$. We determine $v_{0}=133 \pm 14 \mathrm{~km} \mathrm{~s}^{-1}$ and $r_{0}=5.5 \mathrm{kpc}$ by fitting equation (5) to the $\mathrm{PN}$ major axis rotation curve. No effort is made to correct for the inclination of the galaxy since we are seeing it edge-on (Hui 1992).

2.2.1 Central Mass-to-light Ratio We first assume that the luminous matter is selfgravitating. The scale length of the mass distribution is determined by the effective radius of de Vaucouleurs' law. Because $r_{e}=5.18 \mathrm{kpc}$, we have $a=2.85 \mathrm{kpc}$. The only free parameter in the model, $\mathrm{M} / \mathrm{L}$, is then adjusted to fit the velocity dispersion inside $1.5 \mathrm{kpc}$. The total mass resulting from the best fit is $M_{l}=1.56 \pm 0.04 \times 10^{11} M_{\odot}$. Adopting the luminosity of the galaxy to be $B_{0}=6.71$, the mass-to-light ratio is $M / L_{B}=3.9$. The value follows the M/L - L relationship comfortably (Figure 7, Kormendy 1987) for the galaxy's absolute magnitude $M_{B}=-20.80$. Kormendy argued that the dark matter is probably negligible in the center of elliptical galaxies as implied by the $\mathrm{M} / \mathrm{L}-\mathrm{L}$ relation. The fact that the M/L ratio of NGC 5128 follows the general relationship suggests that dark matter is of minor importance in the inner region. The predicted velocity dispersion is plotted in Figure 4 as dashed line. Although the fit is excellent in the central region (upper panel), in the halo the model decreases much faster than the observed PN velocity dispersion (lower panel).

2.2.2 Halo Mass Distribution We adopt the two component mass model of equation (4), which has four parameters: two scale lengths, the total luminous mass $M_{l}$ and the parameter $M_{d}$. While the scale length of the luminous component is set by de Vaucouleurs' law, the others are determined by fitting the model to the combined velocity dispersion profile of the long slit spectra in the inner $1.5 \mathrm{kpc}$ and the PN data between 2 and $22 \mathrm{kpc}$. The best fitting model yields $M_{l}=1.24 \times 10^{11} M_{\odot}, M_{d}=9.49 \times 10^{11} M_{\odot}$, and $d=27.5 \mathrm{kpc}$. The total luminous mass here is smaller than the value $M_{l}=1.56 \times 10^{11} M_{\odot}$ derived in the self-consistent model. This is because we allow the dark mass density to vary continuously towards the center of the galaxy so that it does not have a hollow core. The total mass distribution, its two components, and the mass-to-light ratio are plotted in Figure 5. 

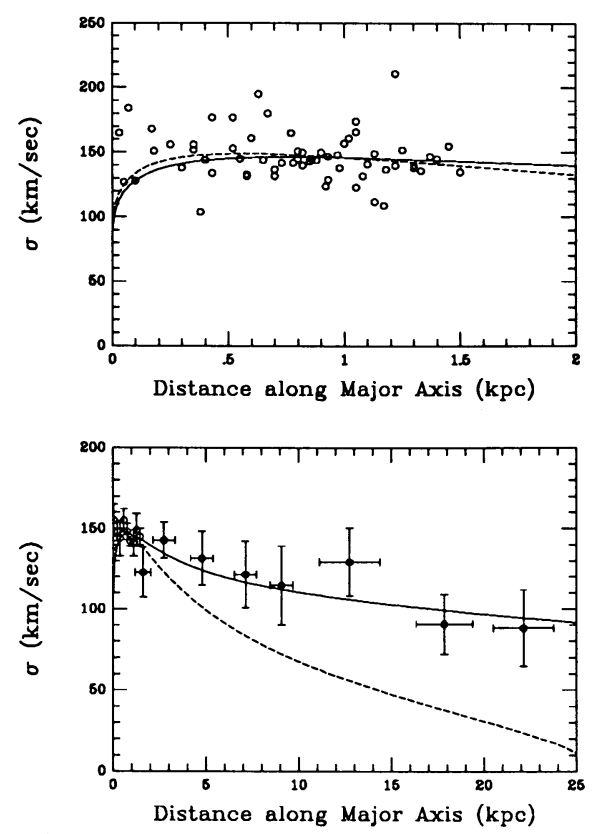

Figure 4. Comparison between the models and the velocity dispersion of integrated light (upper panel) and of planetary nebulae (lower panel). The dashed line is for the selfconsistent model and the solid line is for the model with dark matter.

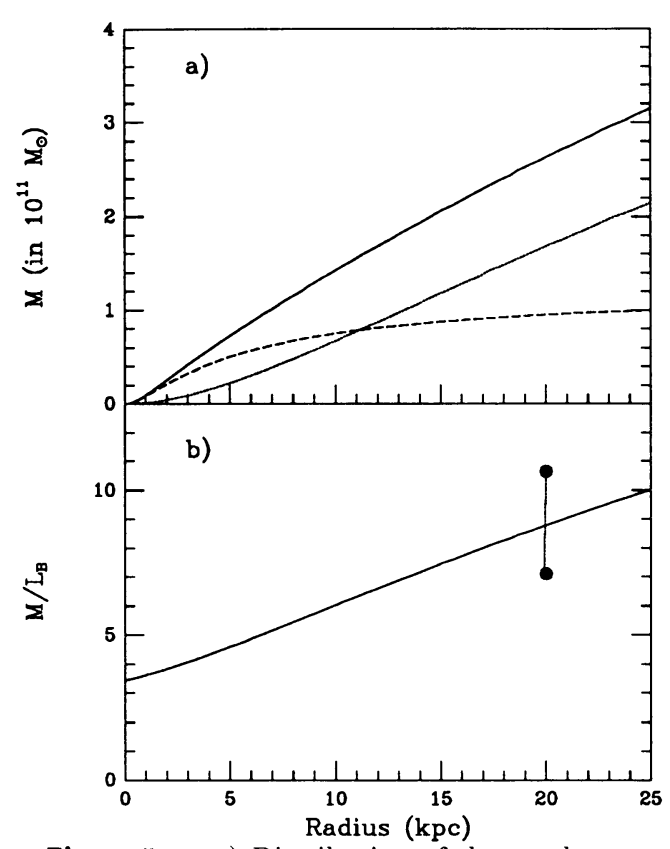

Figure 5. a) Distribution of the total mass (solid curve), the luminous component (dashed curve) and the dark component (dotted curve) as a function of radius. b) Mass-to-light ratio. The two dots linked by a dotted line are $M / L_{B}$ derived using globular clusters.

Within $25 \mathrm{kpc}$, the total mass of the galaxy is $3.1 \times 10^{11} M_{\odot}$, and $M / L_{B}=10$. The model velocity dispersion is shown in Figure 4 as the solid line.

We used the projected mass estimator (Heisler, Tremaine \& Bahcall 1985) to calculate the total mass of the galaxy within $20 \mathrm{kpc}$ from 81 globular cluster velocities (Harris et al. 1988; Sharples 1988). The mass is $3.6 \times 10^{11} M_{\odot}$ if the GCs are on isotropic orbits, or $2.4 \times 10^{11} M_{\odot}$ if they are on tangential orbits. The two dots linked by a dotted line in Figure $5 \mathrm{~b}$ are the corresponding mass-to-light ratios. These two values bracket the mass-tolight ratio given by the PN velocity dispersion. This result suggests that globular clusters are on partially tangential orbits. This is not surprising given the fact that globular clusters beyond $10 \mathrm{kpc}$ are identified by their slightly fuzzy appearance. The resulting sample thus consists of extended and bright globular clusters almost exclusively. These clusters are expected to be on low eccentricity orbits which result in little tidal stripping. 


\section{Current and Future Works}

Planetary nebula systems are under study in more distance galaxies. We have made an extensive PN survey of NGC 4594, the Sombrero galaxy (Ford et al. 1992). Over $300 \mathrm{PNe}$ were identified in the galaxy to a radius of $18 \mathrm{kpc}$. In collaboration with K. Freeman, velocities of $150 \mathrm{PNe}$ were measured on AAT. Detailed dynamical study is underway. Similar work has been carried out on NGC 3379 by Ciardullo and Jacoby (this volume). Interestingly, they found that the PN velocity dispersion decreases to $65 \mathrm{~km} \mathrm{~s}^{-1}$ at about $3 r_{e}$ in good agreement with a constant mass-to-light ratio model. Both work demonstrate that $\mathrm{PN}$ velocities can be measured to at least $10 \mathrm{Mpc}$ with $4-\mathrm{m}$ telescopes. With the new generation telescopes of $8-\mathrm{m}$ or $10-\mathrm{m}$ class, one can expect to apply the same method to galaxies in the Virgo Cluster and beyond. A significant increase in the sample size promises to make important contributions to the dynamical studies of early type galaxies.

\section{Reference}

Binney, J. 1985, M.N.R.A.S., 212, 767

Bland, J., Taylor, K., \& Atherton, P.D. 1987, M.N.R.A.S., 228, 595

Ciardullo, R., Jacoby, G.H. \& Ford, H.C. 1989, Ap. J., 344, 1989

Ciardullo, R., Jacoby, G.H. \& Harris, W.E., 1991, Ap. J., 383, 487

Dubinski, J \& Carlberg, R.G. 1991, Ap. J., 378, 496

Dufour, R.J., van den Berge, S., Harvel, C.A., Martins, D.H., Schiffer, III, F.H., Talbot, Jr., R.J., Talent, D.L. \& Wells., D.N. 1979, A. J., 84, 284

Ford, H.C., Hui, X., Ciardullo, R., Jacoby, G.H. \& Freeman, K.C. 1992, submitted to Ap.J

Graham, J.A. 1979, Ap. J., 232, 60

Harris, H.C., Harris, G.L.H., \& Hesser, J.E. 1988, in IA U Symposium 126, Globular Cluster Systems in Galaxies, eds. J.E. Grindlay \& A.G.D. Philip, (Kluwer Academic), p 205

Heisler, J., Tremaine, S. \& Bahcall J.N. 1985, Ap. J., 298, 8

Hernquist, L. 1990, Ap. J., 356, 359

Hui, X. 1992, Ph.D Thesis, Boston University

Hui, X., Ford, H.C., Ciardullo, R. \& Jacoby, G.H. 1992a, submitted to Ap.J

Hui, X., Ford, H.C., Freeman, K.C. \& Dopita, M.A. 1992b, in preparation

Jacoby, G.H., Ciardullo, R. \& Ford, H.C. 1990, Ap. J., 356, 332

Kormendy, J. 1987, in IA U Symposium No. 127, Structure and Dynamics of Elliptical Galaxies, ed. T. de Zeeuw (Dordrecht: Reidel), p17

Nicholson, R.A., Bland-Hawthorn, J. \& Taylor, K. 1992, Ap. J., $\mathbf{3 8 7 , 5 0 3}$

Sandage, 1961, in The Hubble Atlas of Galaxies

Sharples, R.M. 1988, in IAU Symposium 126, Globular Cluster Systems in Galaxies, eds. J.E. Grindlay \& A.G.D. Philip, (Kluwer Academic), p 545

Stark, A.A. 1977, Ap. J., 213, 368

Statler, T.S. 1987, Ap. J., 321, 113

van Gorkom, J.H., van der Hulst, J.M., Haschich, A.D. \& Tubbs, A.D., 1990, A. J., 99, 1781

Wilkinson, A., Sharples, R.M., Fosury, R.A.E. \& Wallace, P.T. 1986, M.N.R.A.S., 218, 297 